\title{
O papel da educação interprofissional no processo de reorientação da formação em saúde
}

\author{
Edlaine Faria de Moura Villela ${ }^{1}$, Thania Maria Diniz' ${ }^{1}$, Bruno Rodrigues \\ Ferreira1, Maria Gisélia da Silva Rocha', Leilineia Pereira Ramos de \\ Rezende Garcia ${ }^{1}$ e Tamara Rodrigues Lima Zanuzzi ${ }^{1}$ \\ ${ }^{1}$ Programa de Pós-Graduação em Saúde Coletiva do Instituto de Patologia Tropical e Saúde \\ Pública da Universidade Federal de Goiás, Brasil | edlaine@alumni.usp.br; \\ thanymaria@yahoo.com.br; brunorodriferreira@hotmail.com; gilmaryy@hotmail.com; \\ leilineiagarcia@gmail.com; tamaralima8585@gmail.com | https://orcid.org/0000-0002-7043- \\ 2007; https://orcid.org/0000-0001-7014-7719; https://orcid.org/0000-0002-0203-2781; \\ https://orcid.org/0000-0001-7821-1594; https://orcid.org/0000-0002-1187-1376; \\ https://orcid.org/0000-0002-2551-0483
}

Resumo: Introdução: No Brasil, nos últimos anos, vem sendo verificado um esforço para incorporar a educação interprofissional nas políticas indutoras de reorientação profissional em saúde. Nesse contexto, destaca-se o Programa de Educação pelo Trabalho para a Saúde, fundamentado na tríade ensino-serviço-comunidade. Objetivos: Descrever experiências e conhecimentos construídos coletivamente na implementação do PET-I no contexto das práticas da educação interprofissional no município de Jataí, Goiás. Métodos: Estudo descritivo exploratório, de abordagem qualitativa, fundamentado nas bases teóricoconceituais e metodológicas da educação interprofissional, realizado entre agosto de 2019 e novembro de 2020. Resultados: Todos os participantes de cada etapa da pesquisa apresentaram uma atitude positiva e receptiva em relação ao trabalho interprofissional, mencionando que ele promove resultados benéficos, especialmente para a resolutividade da atenção e da segurança dos usuários. Em alguns momentos, porém, verificaram-se visões estereotipadas sobre as profissões. Foi marcante o pensamento de que a EIP é algo muito bom, que apresenta vantagens, mas é muito difícil fazê-la e conseguir disseminá-la para todos os profissionais ou trabalhar de fato em equipe. Conclusões: Ao analisar as percepções dos participantes percebemos que as habilidades e conhecimentos propostos pelo PET-I para uma formação interprofissional podem superar a mecanização e as visões fragmentadas do cuidado em saúde, resultando em uma formação humanizada, integrada e que pode gerar novas respostas às atuais necessidades.

Palavras-chave: Aprendizagem Baseada em Problemas; Equipe de Saúde; Formação Profissional em Saúde; Relações Interprofissionais.

\section{The Role of Interprofessional Education in the Process of Reorientation of Health Training}

Abstract: Introduction: In Brazil, in recent years, an effort has been verified to incorporate interprofessional education into policies that induce professional reorientation in health. In this context, the Program of Education through Work for Health stands out, based on the triad of teaching-service-community. Objectives: To describe experiences and knowledge collectively constructed in the implementation of PET-I in the context of interprofessional education practices in the municipality of Jataí, Goiás. Methods: Descriptive exploratory study, with a qualitative approach, based on the theoretical-conceptual and methodological bases of interprofessional education, carried out between August 2019 and November 2020.Results: All participants of each stage of the research presented a positive and receptive attitude towards interprofessional work, mentioning that it promotes beneficial results, especially for the resolution of attention and safety of users. At times, however, there were stereotyped views about the professions. It was remarkable the thought that IPE is something very good, which has advantages, but it is very difficult to do it and be able to disseminate it to all professionals or actually work as a team. Conclusions: When analyzing the participants perceptions, we realized that the skills and knowledge proposed by PET-I for interprofessional training can overcome mechanization and fragmented views of health care, resulting in a humanized, integrated training that can generate new responses to current needs.

Keywords: Problem-based Learning; Patient Care Team; Health Human Resource Training Internacional Relation. 


\section{Introdução}

A educação interprofissional (EIP) é uma abordagem educacional em que atores de duas ou mais profissões de saúde têm a oportunidade clara e intencional de aprender em conjunto, de forma interativa, visando a estimular e reforçar práticas colaborativas e qualificar os serviços ofertados (Reeves, 2016).

Estudos apontam o potencial da EIP para qualificar a vivência dos usuários no cuidado (Reeves, 2016), bem como a prática profissional, ampliando a satisfação dos trabalhadores (Peduzzi et al., 2020), sendo fundamental para dissipar estereótipos e preconceitos (Visser et al., 2018). Em parte, esse potencial é pertinente ao seu arcabouço teórico-conceitual e metodológico, comprometido com a construção de processos educativos para profissionais de saúde mais capacitados e propícios para trabalhar em equipe e, consequentemente, para a consolidação do SUS (Reeves, 2016; Peduzzi et al., 2013).

Para que as mudanças na formação inicial e nas intervenções dos profissionais atuantes na saúde sejam factíveis, também é necessário o domínio de saberes acerca dos determinantes do processo saúde-doença como produto da interação de vários saberes. Assim, os currículos de formação dos profissionais de saúde devem contemplar não só o conhecimento técnico-científico, mas também enfatizar preceitos éticos, humanos e sociais propostos pela saúde coletiva, valorizar a interdisciplinaridade, o desenvolvimento de competências para as relações sociais, problematizar a realidade para a efetivação dos serviços de saúde de qualidade conforme as necessidades da população, sem repetir o modelo tradicional, biomédico e fragmentado (Mendes et al., 2017).

No Brasil, nos últimos anos, vem sendo verificado um esforço para incorporar a educação interprofissional nas políticas indutoras de reorientação profissional em saúde (Reeves, 2016; Reeves et al., 2016). Nesse contexto, destaca-se o Programa de Educação pelo Trabalho para a Saúde (PET-Saúde), fundamentado na tríade ensino-serviçocomunidade, que tem o objetivo de desenvolver grupos de estudos tutoriais nos campos estratégicos do SUS (Brasil, 2008). Assim, o PET-Saúde busca otimizar os programas de qualificação e valorização dos profissionais de saúde e professores, bem como a iniciação ao trabalho, os estágios e as experiências direcionadas aos alunos da área, segundo as demandas do SUS. Baseia-se na indissociabilidade entre ensino, pesquisa e extensão e integração das comunidades no ensino-serviço (Santos \& Batista, 2018; Haddad et al., 2012).

Desse modo, o PET-I reforça a estratégia educacional como instrumento de melhoraria da qualidade da atenção e de aprimoramento da formação profissional em saúde pelas instituições de ensino e serviços de saúde na perspectiva do SUS (Brasil, 2018). Nessa perspectiva é recomendada a integração e inserção da força de trabalho em cenários de aprendizagem que visem à assimilação da teoria com a prática e do ensino com o serviço. Um ponto relevante para a educação interprofissional é assegurar um contexto de prática e aprendizado que permita ao participante conhecer as outras profissões e desenvolver qualidades: disponibilidade, prática centrada no usuário, confiança nos outros, ser diplomático, autêntico e aberto aos diálogos (Flood et al., 2019). Sordi et al. (2020) reforçam o potencial de alianças realizadas entre as IES com os serviços de saúde locorregionais como uma medida para superar a fragmentação entre teoria e prática, pois elas trazem mudanças nos cenários de aprendizagem e no modelo de formação das práticas profissionais em saúde. 
Ao aderir ao PET-I, a Universidade Federal de Jataí (UFJ) iniciou um processo de inserção da EIP em projeto curricular, estimulando o trabalho em equipe referenciado nas diretrizes operacionais de reestruturação do SUS, saindo de fato do status de recomendação para ação efetiva assentada nas práticas. Com essa iniciativa o campus propicia condições para que docentes, discentes, preceptores e a rede de saúde jataiense possam trabalhar de forma integrada, sistematizada e colaborativa. Assim, justifica-se a realização desse estudo com o intuito de descrever experiências e conhecimentos construídos coletivamente entre docentes coordenadores, docentes tutores, preceptores de diferentes profissões de saúde e discentes de graduação na área de saúde da UFJ, na implementação do PET-I no contexto das práticas da educação interprofissional no município de Jataí, Goiás.

\section{Desenvolvimento do Estudo}

\subsection{População e Período de Estudo}

Este estudo é descritivo exploratório, de abordagem qualitativa, fundamentado nas bases teórico-conceituais e metodológicas da EIP, e foi realizado entre agosto de 2019 e novembro de 2020, no município de Jataí. A natureza qualitativa permite explorar o fenômeno a partir do significado que as pessoas atribuem a suas experiências de vida; compreende a subjetividade, valores, percepções e o modo como elas entendem e constroem o mundo ao seu redor (Vieira \& Hossne, 2015). Mais que isso, como os indivíduos evoluem e se transformam a cada dia (Andrade \& Pegolo, 2020).

A equipe foi composta por participantes do programa PET-I da Universidade Federal de Jataí (UFJ) que agregava docentes coordenadores, docentes tutores, preceptores de diversas profissões de saúde e discentes de graduações das áreas de saúde da UFJ. O programa PET-I da UFJ tinha o envolvimento dos cursos de Biomedicina, Educação Física, Enfermagem, Fisioterapia e Medicina. O grupo analisado nesta pesquisa era composto por doze integrantes, sendo um estudante do curso de Medicina, dois do curso de Enfermagem, dois do curso de Fisioterapia e um de Educação Física. Entre as quatro preceptoras, duas eram psicólogas, uma enfermeira e a outra fisioterapeuta. Também havia uma docente coordenadora (professora da Biomedicina) e uma docente tutora (professora da Medicina). Esse subgrupo foi intitulado "Saúde \& Interdisciplinaridade", e realizou 16 encontros com atividades diversas.

\subsection{Coleta e Análise dos Dados}

\subsubsection{Coleta de Dados}

Para a organização da formação do grupo de participantes e para coleta de dados foram realizados 35 encontros com um total de 60 participantes compostos por docentes, discentes e trabalhadores da área da saúde. 
Os encontros eram realizados quinzenalmente, sempre às sextas-feiras, onde eram propostas atividades diversas como: reuniões gerais com os 60 integrantes, atividades de definição do plano de trabalho, aulas sobre capacitação em EIP em saúde, palestra ("A importância da interprofissionalidade no atendimento à saúde e a qualidade de vida dos discentes"), atividades de extensão em instituições e em contato com a comunidade (Hepatite Zero, palestras e educação em saúde individual, aproximação com a comunidade e os serviços de saúde), cursos de capacitação sobre acolhimento em saúde, eventos e apresentações sobre estudos da EIP, participação e apresentação de trabalhos no CONEPE (Congresso de Ensino, Pesquisa e Extensão da UFJ), organização de evento sobre abordagens interprofissionais em saúde coletiva- gastroenterites, visitas as unidades básica em saúde (UBS), atividades de estudo sobre métodos para a análise e cogestão de coletivos, rodas de conversas para apresentação de materiais produzidos nos eventos e intervenções na comunidade.

A partir dos encontros com o grupo geral, foi constituído um subgrupo composto por 12 participantes do PET-I de Jataí, sendo um estudante do curso de Medicina, dois do curso de Enfermagem, dois do curso de Fisioterapia e um de Educação Física. Entre as quatro preceptoras, duas eram psicólogas, uma enfermeira e a outra fisioterapeuta. Também havia uma docente coordenadora (professora da Biomedicina) e uma docente tutora (professora da Medicina). Esse subgrupo foi intitulado "Saúde \& Interdisciplinaridade", e realizou 16 encontros com atividades diversas, contemplando também as atividades do grupo geral com 60 participantes.

Para a coleta de dados para esta pesquisa o grupo "Saúde \& Interdisciplinaridade" composto por 12 participantes foram informados sobre os objetivos desta pesquisa, concordando com eles voluntariamente por meio do TCLE que foi lido, esclarecido e assinado por todos, antes do início das atividades de coleta, que seriam atividades com grupos focais e análise documental. Para tanto, foi solicitado o uso de gravador para registrar o conteúdo das discussões, assegurando-se aos participantes o respeito à confidencialidade e ao anonimato. As conversas foram transcritas de modo literal para a posterior análise de dados.

O estudo com o grupo "Saúde \& Interdisciplinaridade" foi realizado em três fases, cada uma delas com procedimento de coleta de dados específicos. A primeira delas, antes da imersão dos participantes nas atividades do PET-I Jataí, consistiu em um grupo focal (GF1), cujo objetivo era conhecer os saberes prévios dos participantes acerca da EIP e do trabalho em equipe. A segunda foi a análise documental das atividades que ocorreram durante a execução do PET-I, por meio da coleta dos portfólios e registro das atividades remotas, para coleta das experiências, percepções e reflexões dos participantes. A terceira fase foi a realização do segundo grupo focal (GF2), convocado remotamente, após as intervenções previstas para a equipe. Seu objetivo foi verificar as mudanças dos conhecimentos, experiências e percepções após um ano de projeto. A coleta de dados, desde a primeira até a última etapa, foi realizada entre agosto de 2019 e novembro de 2020.

\subsubsection{Grupo Focal 1 (GF1)}

O Grupo Focal em seu caráter subjetivo de investigação é utilizado como estratégia metodológica qualitativa, e caracteriza-se por buscar respostas acerca do que as pessoas pensam e quais são seus sentimentos. O método qualitativo é utilizado para estudar temas específicos em contextos coletivos. O grupo focal é um meio de compreensão das percepções, atitudes, interpretações, crenças e representações sociais sobre questões comuns. Sendo assim, é um conjunto de pessoas com características ou experiências similares, que se baseia na comunicação, na interação e nas impressões de seus participantes para criar um ambiente favorável à discussão (Mazucato, 2018). Esse recurso possibilitou aos participantes da presente pesquisa manifestar percepções e pontos de vista sobre saberes e vivências em ações na perspectiva da EIP e sobre o trabalho em equipe. 
O convite para o encontro foi enviado aos 12 integrantes, por meio eletrônico, pela tutora do grupo "Saúde \& Interdisciplinaridade". Todos foram cadastrados no início do programa em um grupo de comunicação eletrônica, sendo este o principal meio de comunicação entre eles.

\subsubsection{Análise Documental}

Para Bardin (2011) a análise documental tem o objetivo de analisar e representar de forma condensada as informações provenientes dos elementos pesquisados, que permitem elaborar um documento secundário com o máximo de informações pertinentes sobre a temática em foco. A coleta de dados através da análise documental com o uso dos portfólios (é o nome que se dá a um conjunto de tarefas realizadas durante um período de tempo) considera as características individuais e singulares dos indivíduos e destaca as competências desenvolvidas por cada um, que escolhe o material a ser incluído. Contêm suas opiniões, dúvidas e reações aos conteúdos estudados, assim como suas reflexões sobre o desenvolvimento da própria aprendizagem (Cotta \& Costa, 2016). Os portfólios do presente estudo, recolhidos e fotografados pela pesquisadora e arquivados para análise, contêm as práticas realizadas pelos participantes no período de setembro de 2019 à março de 2020, quando a UFJ suspendeu o ensino presidencial em razão da pandemia de covid19. Após esse período, a realização das atividades foi virtual, passando a coleta dos dados a ser realizada nesses meios. Essa análise documental, baseada no portfólio, foi um registro inicial das ações do PET-I durante e após um ano do projeto, o que permitiu uma avaliação longitudinal e mais fidedigna (Cotta \& Costa, 2016). Foram recolhidos seis portfólios para análise e estudo dos dados.

$\mathrm{Na}$ análise documental foram abordadas as atividades do grupo "Saúde \& Interdisciplinaridade" nos serviços de saúde e na UFJ que primavam pela participação, pela interação e pelo respeito entre os seus integrantes, bem como aquelas que promoviam a reflexão e o pensamento crítico com foco no desenvolvimento de competências para o trabalho em equipe. As estratégias utilizadas pelo grupo foram construídas longitudinalmente, a partir dos encontros, nos quais eram discutidas as necessidades, as dificuldades e o interesse de todos nos temas debatidos.

Inicialmente, foi feito um levantamento dos conhecimentos e das experiências anteriores dos participantes do Grupo em EIP, seguido de uma aprofundação dos elementos teóricos e práticos da abordagem. Desse modo, buscou-se um alinhamento dos principais conceitos que permitiu eliminar dúvidas e incertezas sobre o assunto. Como o tema do PET-I Jataí é o acolhimento, também foi realizado um estudo teórico sobre essa questão e, houve muitas atividades envolvendo as metodologias ativas. Além disso, foram feitas muitas leituras, observações e rodas de conversa para a discussão de percepções e vivências. O contato com os usuários e a comunidade não foi muito explorado, nem mesmo antes da pandemia, porque a Secretaria de Saúde de Jataí impunha limitações à atuação dos alunos da UFJ nos serviços de saúde municipais. Em geral, essas atividades ocorreram em ações de educação, e não de clínica, em praças, num shopping e no Centro de Referência de Assistência Social (CRAS). Ainda assim, os integrantes puderam desenvolver competências colaborativas e aprender uns com os outros.

Paralelamente, ocorriam outras dinâmicas com vistas a possibilitar que os integrantes se sentissem à vontade para conhecer uns aos outros e, principalmente, esclarecer dúvidas sobre suas profissões, seus papéis e suas funções. A leitura, a análise e a posterior discussão de artigos com essas dinâmicas, mapas mentais, teatro, rodas de conversa e murais permitiram a aquisição de novos conhecimentos e o desenvolvimento de uma boa interação, proporcionando um clima favorável e receptivo ao desenvolvimento do trabalho colaborativo e à interação profissional. 
Especificamente, a dinâmica do barquinho - na qual o grupo tinha de escolher um líder e, em equipe, construir um barquinho com quatro folhas de papel-sulfite e fita adesiva, sendo que só era permitida a comunicação por gestos e apenas nos três minutos finais era possível conversar para realizar a atividade - permitiu a compreensão da importância da comunicação entre os membros de uma equipe para a obtenção de melhores resultados do grupo.

Os vários cursos on-line contribuíram para o alinhamento conceitual do PET-I Jataí, mas foram as webconferências as iniciativas de maior relevância, especialmente durante a pandemia. Elas possibilitaram o "contato" virtual com pessoas, profissionais e instituições de todo o mundo, momentos de imensa troca de conhecimentos e experiências entre os diferentes projetos PET-I no país. As dinâmicas no Google Meet, no Zoom e no Google Classroom promoveram uma qualificação dos participantes no uso de ferramentas e tecnologias digitais, o que lhes possibilitou diversas atividades interativas e participativas, que lhes trouxeram um pouco de leveza no meio da pandemia e promoveram um intenso diálogo sobre a EIP na saúde.

\subsubsection{Grupo Focal 2 (GF2)}

Os avanços nas tecnologias de informação e comunicação, bem como a facilidade de acesso a recursos digitais, oferecem novas oportunidades para a realização de pesquisas qualitativas. Assim, quando o mundo foi surpreendido pela pandemia, o contexto de distanciamento social lançou um enorme desafio a ser superado para que as pesquisas não fossem interrompidas. Nesse contexto, emergiu a ideia de um grupo focal on-line, obedecendo ao mesmo rigor metodológico observado em grupos focais presenciais (Mello \& Cruz, 2018).

Como já havia acontecido no primeiro encontro, o convite para a reunião virtual foi enviado para os integrantes do grupo "Saúde e Interdisciplinaridade" através de mensagens eletrônicas. O encontro virtual, realizado via Google Meet, buscava identificar mudanças nos conhecimentos, nas habilidades e nas competências dos integrantes.

\subsubsection{Análise dos Dados}

Buscando conhecer a vivência dos docentes e estudantes que participaram da pesquisa, bem como suas percepções e seus saberes sobre educação interprofissional na experiência PET-I em Jataí, utilizou-se a análise de conteúdo de Bardin (2011), tendo também como base o referencial teórico-conceitual e metodológico da EIP. A análise temática facilita a compreensão da linguagem dos participantes ao relacionar a significação dos termos descritos aos temas essenciais do fenômeno investigado (Minayo, 2014), articulando o discurso com o contexto de produção (Bardin, 2011).

Nessa análise, buscou-se compreender as características, estruturas ou modelos que estão por trás dos fragmentos de mensagens discutidas sobre o papel da educação interprofissional no processo de reorientação da formação em saúde. Durante a interpretação dos dados, foi realizada de forma criteriosa a descrição dos resultados e transcrição das respostas das questões norteadoras discutidas nos encontros grupais. A relação entre os dados obtidos e a fundamentação teórica da EIP, foram as bases estruturais da análise dos dados. 


\section{Aspectos Éticos}

O presente estudo foi aprovado pelo Comitê de Ética em Pesquisa (CEP) da UFJ, por meio do parecer de no 3.727.135, respeitando-se os princípios éticos da pesquisa com seres humanos (autonomia, beneficência, não maleficência, justiça e equidade), de acordo com a Resolução 466, de 12 de dezembro de 2012.

Em razão da pandemia, o estudo foi aprovado pelo CEP uma segunda vez, para aprovação da realização do Grupo Focal 02 de forma remota.

\section{Resultados e Discussão}

\subsection{Grupo Focal 1 (GF1)}

A ação foi iniciada com a apresentação de cada participante. Ao longo do encontro, buscou-se manter um clima agradável, informal, mas sem perder o foco no planejamento. O encontro teve duração de 1 hora e 30 minutos. Com base no referencial teórico da EIP, foi elaborado um roteiro para a condução do grupo focal, composto de questões-chave baseadas no objetivo da pesquisa. A discussão foi desencadeada por uma questão norteadora para desdobramento do problema: "Por que aprendemos separados se depois trabalharemos juntos?". O intuito foi problematizar a realidade dos serviços de saúde e levantar temas pertinentes ao processo de formação em saúde na perspectiva da EIP. Em seguida, foi perguntado aos participantes o que eles entendiam por educação interprofissional, seus efeitos e sua realização prática; o que eles entendiam por trabalho em equipe, como seria feito, seus pontos fortes e fracos. Depois, buscou-se conhecer as experiências prévias do grupo, saber se alguém já tinha trabalhado em equipe ou experimentado uma aprendizagem compartilhada e verificar seus resultados, estratégias e expectativas de aprendizado ao se inscreverem no PET-I.

Os objetivos desse grupo focal foram descobrir se os integrantes conheciam a EIP (se já haviam tido experiência prática integrada) e identificar seus conhecimentos sobre o trabalho em equipe e suas percepções e expectativas acerca da temática e do PET-Jataí.

Compreendeu-se nos relatos que todos os participantes têm uma atitude positiva e receptiva em relação ao trabalho interprofissional, mencionando que ele promove resultados benéficos, especialmente para a resolutividade da atenção e da segurança dos usuários. Em alguns momentos, porém, verificaram-se visões estereotipadas sobre as profissões. Foi marcante o pensamento de que a EIP é algo muito bom, que apresenta vantagens, mas é muito difícil fazê-la e conseguir disseminá-la para todos os profissionais ou trabalhar de fato em equipe. No entanto, quando questionados sobre as expectativas, todos reponderam que eram muito positivos e esperançosos de que se conseguissem bons resultados com o PET-I.

Assim, os integrantes perceberam a necessidade de pensar mudanças nessa educação a partir da discussão da articulação entre as universidades e os serviços de saúde para também favorecer a compreensão das necessidades de saúde da população e direcionar essa educação para o SUS, que seria beneficiado com a inserção desses futuros profissionais nos serviços. 


\subsection{Análise Documental}

Em um segundo momento, foram analisados os portfólios, uma estratégia de avaliação individual de cada estudante. Mas também os preceptores construíram seus portfólios, registrando seus conhecimentos e percepções como oportunidade de aprendizado. As docentes tutoras acompanharam a evolução de todos os participantes, observando os apontamentos de seus portfólios, em que eles fizeram uma anotação diária das atividades das quais participaram, com seu entendimento quanto às ações de aprendizagem compartilhada.

Todas as atividades registradas nos meios digitais, no período de abril de 2020 até o mês de novembro de 2020 , foram impressas ou tiveram recolhidas sua gravação para análise. A interpretação dos dados evidenciou a importância do trabalho em equipe e construção coletiva; evidenciando a equidade em saúde e o olhar sob novas práticas culturais. As estratégias de ensino na universidade e nos serviços de saúde e seu papel na formação em saúde, também foram aspectos exaltados nas discussões, ressaltando de forma crítica a escassez de relatos de atividades práticas no serviço de saúde.

É fundamental que essa aprendizagem seja reforçada por metodologias que produzam conhecimentos e competências para o trabalho em equipe e, também, que promovam a colaboração (Peduzzi, 2016; Reeves, 2016).

\subsection{Grupo Focal 2 (GF2)}

O GF2 foi conduzido com os mesmos integrantes do GF1 (grupo "Saúde \& Interdisciplinariedade"). Os participantes tiveram uma percepção positiva de sua experiência no projeto, levando em consideração as atividades realizadas tanto no grupo focal 1 e na análise documental. Eles ressaltaram, em suas falas, a relevância da experiência do trabalho interprofissional, do convívio com pessoas de diferentes formações e do aprendizado, oportunizados pelo PET-I, para a formação profissional em saúde. Mencionaram que, com as atividades realizadas no âmbito do projeto, passaram a se sentir agentes de mudança. Relataram que tiveram a oportunidade de conhecer mais o trabalho e campo profissional dos colegas.

No que diz respeito à EIP e o trabalho em equipe, percebeu-se um amadurecimento sobre o entendimento da EIP, diante da melhoria de suas habilidades com o trabalho interprofissional. As necessidades de saúde no contexto atual são muitas e dinâmicas; os participantes reconheceram o seu caráter complexo e defenderam a importância dos vários atores no trabalho em saúde, como relatado nos depoimentos a seguir:
"A EIP é muito importante para melhorar a colaboração. A interprofissionalidade aumenta a qualidade da assistência aos pacientes. Assim, a EIP se mostra uma oportunidade de trocar conhecimentos, ampliar olhares, e projetar novos caminhos para a formação de profissionais de saúde melhor preparados para práticas compartilhadas"

A lógica da formação em saúde ainda está muito voltada para as especificidades de cada área, sendo negligenciada, ainda, a formação de competências para o trabalho em equipe com base na colaboração. Além disso, os desenhos curriculares ou programas dos componentes curriculares não têm demonstrado a intencionalidade de viabilizar momentos de aprendizagem comuns a diversos cursos.

Por outro lado, foi evidenciado que a educação interprofissional também provoca resistência, na academia e no serviço, visto que incentiva a mudança de hábitos na busca por algo novo e desconhecido para a maioria dos envolvidos. $E$ também porque esse movimento tenta romper com a cultura individualista na área da saúde. A maior barreira para a consolidação da interprofissionalidade na prática é o desconhecimento acerca do tema, em especial entre aqueles já formados e que não tiveram contato com a temática. Estes são mais relutantes ao debate sobre estereótipos, hierarquias e compartilhamento colaborativo, principalmente ao trabalho em equipe. 
Diante do exposto, observa-se a necessidade de planejar muito bem a abordagem a ser executada, para que ocorra de forma interativa, significativa e compartilhada, com claro objetivo de desenvolver a colaboração entre os participantes (Barr \& Low, 2013). Para Reeves et al. (2016), não se aprende a trabalhar em equipe colaborativamente apenas fazendo o trabalho em saúde por si.

\section{Conclusões}

Os resultados da pesquisa apontam para uma realidade que dá ênfase à formação de saberes e habilidades uniprofissionais, o que dificulta a ruptura com o modelo tradicional de produção de serviços de saúde. No entanto, neste estudo identificamos que o PET-I pode ser um espaço favorável para concretizar a capacitação e as competências para a formação de um perfil profissional propostas nas Diretrizes Curriculares Nacionais (DCN) para os Cursos da Saúde que objetivam fortalecer o SUS, através de um ensino inovador.

Ao analisar as percepções dos participantes percebemos que as habilidades e conhecimentos propostos pelo PET-I para uma formação interprofissional podem superar a mecanização e as visões fragmentadas do cuidado em saúde, resultando em uma formação humanizada, integrada e que pode gerar novas respostas às atuais necessidades. No entanto, aponta a necessidade de se implementar os fundamentos da EIP, desde o início da graduação para construir conhecimentos além do núcleo profissional e aprimorar a formação do trabalho em equipe, e identificando a comunicação e a troca entre os diferentes saberes como os elementos básicos do trabalho interprofissionale o desenvolvimento de habilidades eficazes de trabalho em equipe, além de recolocar os usuários no centro do processo, para o fortalecimento do sistema de saúde pela melhora dos serviços ofertados.

As ações desenvolvidas mostram que as estratégias pedagógicas adotadas utilizadas foram significativas e criativas, fundamentadas no uso das metodologias ativas e, viabilizando a inclusão dos diferentes saberes profissionais criando oportunidades educacionais nas quais indivíduos de duas ou mais profissões aprendam interagindo uns com os outros, mas com o propósito explicito de avançar na qualificação e desenvolvimento de habilidades e competências para o trabalho em equipe.

\section{Agradecimentos}

A todos os envolvidos no Programa PET Saúde-Interprofissionalidade de Jataí, Goiás.

À Secretaria de Gestão do Trabalho e da Educação na Saúde (SGTES) do Ministério da Saúde e ao Ministério da Educação, que viabilizaram o programa no território brasileiro.

\section{Referências}

Allvin, R., Thompson, C. \& Edelbring, S. (2020). Avaliação da competência interprofissional na educação de profissionais de saúde de graduação: protocolo para uma revisão sistemática de instrumentos de autorrelato. Syst Rev, 9(142). https://doi.org/10.1186/s13643-020-013947

Andrade, S.M.O., \& Pegolo, G.E. (2020). A pesquisa científica em saúde: concepção, execução e apresentação. UFMS.

Bardin, L. (2011). Análise de conteúdo. (3ª ed.) Edições 70.

Barr, H., \& Low H. (2013). Introducing interprofessional education. Fareham: Center for The Advancement of Interprofessional Education. CAIPE.

Brasil. Ministério da Saúde. (2018). Secretaria de Gestão do Trabalho e da Educação na Saúde. Edital $n^{\circ}$ 10, 23 de julho 2018. Seleção para o Programa de Educação pelo Trabalho para a Saúde - PET-SAÚDE/ INTERPROFISSIONALIDADE - 2018/2019. Diário Oficial da União: Brasília, DF. 
Brasil. (2008). Ministério da Saúde e Ministério da Educação. Portaria Interministerial nํ1802, de 26 de agosto de 2008, para fomentar grupos de aprendizagem tutorial na Estratégia de Saúde da Família. http://www.brasilsus.com.br/legislacoes/inter- ministerial/14427-1802.html.

Cotta, R.M.M., \& Costa, G.D. (2016). Instrumento de avaliação e autoavaliação do portfólio reflexivo: uma construção teórico-conceitual. Interface - Comunicação, Saúde, Educação, 20(56), 171-183.

Flood, B., Hocking, C., Smythe, L., \& Jones, M. (2019). Working in a spirit of interprofessional practice: a hermeneutic phenomenological study. Journal of interprofessional care, 33(6), 744-752.

Haddad, A.E., Brenelli, S.F.L., Cury, G.C., Puccini, R.F., Martins, M.A., Ferreira, J.R., \& Campos, F.E. (2012). Pró-Saúde e PET-Saúde: a construção da política brasileira de reorientação da formação profissional em saúde. Revista Brasileira de Educação Médica, 36(1, Suppl. 1), 03 04.

Mazucato, T. (Org). (2018). Metodologia da pesquisa e do trabalho científico. FUNEPE.

Mello, M.G., \& Cruz, L.R. (2018). Documentação metodológica de um grupo focal virtual sobre hackers e ciberpiratas. Acta Scientiarum. Human and Social Sciences, 40(2), e40527.

Mendes, M.C., Lopes, V.C., Souza, H. A., Viana, D.G., \& Bueno, S.V. (2017). Andragogia, métodos e didática do ensino superior: novo lidar com o aprendizado do adulto na EAD. Revista Gestão \& Amp; Saúde, 3(1), 1366-1377. https://periodicos.unb.br/index.php/rgs/article/view/165

Minayo, M.C.S. (2014). O desafio do conhecimento: pesquisa qualitativa em saúde. (14.ed.) Hucitec.

Peduzzi, M. (2016). O SUS é interprofissional. Interface - Comunicação, Saúde, Educação, 20 (56), 199-201.

Peduzzi, M., Agreli, H.L.F., Silva, J.A.M., \& Souza, H.S. (2020). Trabalho em equipe: uma revisita ao conceito e a seus desdobramentos no trabalho interprofissional. Trabalho, Educação e Saúde, 18(Suppl. 1), e0024678.

Peduzzi, M., Norman, I.J., Germani, A.C.C.G., Silva, J.A.M., \& Souza, G.C. (2013). Interprofessional education: training for healthcare professionals for teamwork focusing on users. Revista da Escola de Enfermagem da USP, 47(4), 977-983.

Reeves, S. (2016). Why we need interprofessional education to improve the delivery of safe and effective care. Interface - Comunicação, Saúde, Educação, 20(56), 185-197.

Reeves, S., Fletcher, S., Barr, H., Birch, I., Boet, S., Davies, N., McFadyen, A., Rivera, J., \& Kitto, S. (2016). A BEME systematic review of the effects of interprofessional education: BEME Guide No. 39. Medical teacher, 38(7), 656-668.

Santos, G.M., \& Batista, S.H.S.S. (2018) Docência, Pró-Saúde e PET-Saúde: narrativas de um fazer interprofissional. Interface - Comunicação, Saúde, Educação, 22(Suppl. 2), 1589-1600.

Sordi, M.R.L., Mendes, G.S.C.V., Cyrino, E.G., Alexandre, F.L.F., Manoel, C.M., \& Lopes, C. V.M. (2020). Experiência de construção coletiva de instrumento autoavaliativo a serviço da formação médica referenciada nas Diretrizes Curriculares Nacionais (DCN) pautadas no Programa Mais Médicos. Interface - Comunicação, Saúde, Educação, 24, e190527.

Vieira, S., \& Hossne, W.S. (2015). Metodologia científica para a área da saúde. (2ª ed.) Elsevier.

Visser, C., Wilschut, J.A., Isik, U., Van der Burgt, S.M.E., Croiset, G., \& Kusurkar, R.A. (2018). The Association of Readiness for Interprofessional Learning with empathy, motivation and professional identity development in medical students. BMC medical education, 18(1), 125. 